\title{
STUMP, a surprise finding in a large fibroid uterus in a 20 -year-old woman
}

This article was published in the following Dove Press journal:

International Journal of Women's Health

\section{Lara Hughes \\ Alphonse Roex \\ Anupam Parange}

Obstetrics and Gynecology

Department, The Lyell McEwin

Hospital, Adelaide, SA, Australia
Correspondence: Lara Hughes

Obstetrics and Gynecology Department,

The Lyell McEwin Hospital, Haydown

Road, Elizabeth Vale, SA 5I I2, Australia

Tel +6I 88 I829306

Email lara.hughes@sa.gov.au

\begin{abstract}
We report on a case of a 20-year-old nulliparous woman with menorrhagia associated with a smooth muscle tumor of uncertain malignant potential (STUMP) that was responsive to Goserelin. This case details the investigation and management of a young woman who desires ongoing fertility preservation. STUMP is a rare uterine tumor with a paucity of literature available regarding management and subsequent malignant potential, particularly in the case of a patient who desires fertility preservation.
\end{abstract}

Keywords: smooth muscle tumor of uncertain malignant potential, STUMP, malignant potential, fertility preservation, fibroid uterus

\section{Introduction}

Smooth muscle tumor of uncertain malignant potential (STUMP) encompasses a broad group of uterine neoplasms that do not meet the current histologic criteria for a diagnosis of either benign or a malignant tumor. It is thought that STUMP may represent a "transition" tumor between leiomyoma to leiomyosarcoma or possibly undiagnosed low-grade leiomyosarcoma. ${ }^{1}$ Among women undergoing hysterectomy or myomectomy for a presumed diagnosis of leiomyoma, $0.01 \%$ receive a diagnosis of STUMP. ${ }^{2}$ Due to the rarity of this disease and inconsistency in diagnostic criteria, the true prevalence of STUMP is difficult to determine ${ }^{8}$ Furthermore, there is limited data available on STUMP's malignant potential, and this creates significant controversy surrounding the management, particularly when the subject desires ongoing fertility, such as in the aforementioned case.

\section{Case report}

A 20-year-old nulliparous Cambodian woman was referred to the Lyell McEwin Hospital in Adelaide South Australia by her General Practitioner for assessment of heavy menstrual bleeding on a background of a large fibroid uterus.

She had a history of heavy menstrual periods lasting 8-10 days with clots for the previous 3-4 years. She had reached menarche at 14 years of age and her periods had previously been regular and of normal volume. She had never been sexually active.

She also had recently noticed swelling in her abdomen with symptoms of lower abdominal discomfort, bloating, and increased frequency of micturition. She thought the abdominal swelling had increased rapidly over the previous 5-6 months. There was no associated history of loss of appetite or weight loss.

She had been using the combined oral contraceptive pill for the past year prescribed by her General Practitioner for symptomatic management of her heavy periods. She otherwise had no significant past medical history. 
Examination revealed a slim young female with a body mass index of 21. Her vital signs were normal. Her abdomen was soft and nontender, and there was a firm, irregular mass felt arising from her pelvis that extended $4 \mathrm{~cm}$ above the umbilicus. The mass had ill-defined borders and restricted mobility. There was no evidence of ascites clinically. Pelvic examination was not performed, as the patient was not sexually active.

Her baseline investigations revealed a hemoglobin of $93 \mathrm{~g} / \mathrm{L}$ (reference range $115-155 \mathrm{~g} / \mathrm{L}$ ), normal platelets, low serum ferritin level of $10 \mu \mathrm{g} / \mathrm{L}$ (reference range $30-250 \mu \mathrm{g} / \mathrm{L}$ ), normal lactate dehydrogenase of $163 \mathrm{U} / \mathrm{L}$, normal liver and renal function, and a normal midstream urine culture. Transabdominal ultrasound examination was suggestive of a large subserosal fibroid at the fundus of the uterus measuring $20 \times 18 \mathrm{~cm}$. Both the ovaries could not be imaged as the fibroid was occupying the pelvis, but there was no intraperitoneal free fluid seen.

A provisional diagnosis of heavy menstrual bleeding and iron-deficiency anemia secondary to a large leiomyoma was made. Magnetic resonance imaging (MRI) of the abdomen and pelvis was arranged because of the history of rapid growth.

The MRI using T1/T2 axial coronal and T1 postcontrast imaging confirmed a large fundal fibroid with areas of necrosis, suggestive of intramural leiomyoma with cystic degeneration. There was an associated mild to moderate, right-sided hydronephrosis secondary to pressure from the fibroid. The ovaries appeared normal and there was no associated lymphadenopathy or ascites. The appearance was not suggestive of a malignancy and there was no peritoneal or distant metastasis seen.

The results were discussed with the patient, and after appropriate counseling, the patient was commenced on a $3.6 \mathrm{mg}$ goserelin (gonadotropin-releasing hormone [GnRH] agonist) subcutaneous implant every 4 weeks for 6 months. This drug ultimately results in the suppression of pituitary gonadotropins, resulting in a hypogonadal state in the patient. goserelin is used as a short-term preoperative therapy to facilitate surgical management of fibroid disease by reducing the tumor volume and facilitating improvement in anemia by limiting blood loss.

She tolerated the implant well without any major adverse effects. She was also given an intravenous iron infusion.

After six doses of goserelin, the size of the fibroid had reduced considerably and was now measuring $2 \mathrm{~cm}$ below the umbilicus. Given the fact that the presumed fibroid responded typically with volume reduction to hormonal management, it was interpreted as reassuring for a benign diagnosis. The patient was subsequently consented and booked for a myomectomy. This was performed through a Pfannenstiel incision, revealing an enlarged uterus with a solitary subserosal fibroid in the anterior wall. Both the ovaries and tubes were normal. There was no free fluid in the pelvis and no visible findings suggestive of malignancy. A tourniquet was applied at the level of the isthmus of the uterus in order to temporarily reduce blood flow to the uterus by compressing the ascending branches of the uterine artery and a subsequent myomectomy was performed. The defect was closed in two layers using Polyglactin 910 sutures. Hemostasis was satisfactory and the estimated blood loss was $100 \mathrm{~mL}$. Postoperative recovery was uneventful and the patient was discharged on day 3.

The postoperative histology of the specimen was reported as a STUMP. The tumor had a low mitotic index with five mitotic figures per 10 high-power fields (HPFs) and no coagulative tumor necrosis; however, diffuse significant cytological atypia was reported.

Her case was referred to the Royal Adelaide Hospital's gynecological oncology team and following a multidisciplinary team review along with review of the scientific literature, it was decided that her management should include annual clinical reviews and pelvic ultrasounds and she should have a simple hysterectomy once she completes her family. The patient was reassured regarding the low malignant potential and low risk of recurrence. Written informed consent has been provided by the patient for publication of the case details.

\section{Discussion}

STUMP often presents with symptoms consistent with a benign leiomyoma. As in this case, these symptoms include a combination of abnormal uterine bleeding, pelvic mass, or symptoms due to secondary compression and anemia. ${ }^{3}$ The age of onset of this disease is similar to that of leiomyoma or leiomyosarcoma, and little is known regarding the specific risk factors that predispose to a diagnosis of STUMP. It is suggested in the literature that the mean age at which a diagnosis of STUMP is made is 43 years; however, due to the rarity of this diagnosis, demographic data are limited. ${ }^{4}$ This makes a preoperative diagnosis next to impossible, and hence, STUMP is often unexpectedly found post-hysterectomy or myomectomy. It is very difficult to differentiate between benign leiomyoma and malignant sarcomas preoperatively. MRI has been used to differentiate between benign and malignant tumors of the uterus utilizing increased signal intensity; however, little evidence exists to distinguish STUMP from leiomyoma. ${ }^{5}$ This modality should be used in cases with a high suspicion of a sarcoma, as was the case here where 
the history was suggestive of a rapid increase in the size of the fibroid in a young Asian woman. However, it should be noted that almost all rapidly growing fibroids are histologically benign and MRI cannot reliability exclude malignant pathology. ${ }^{6}$ However, it is the most sensitive imaging modality available to give a preoperative diagnosis of leiomyosarcoma. It has been suggested that $\sim 54 \%$ of uterine leiomyosarcomas were unidentified prior to surgery. ${ }^{7}$

The current criteria for the histopathologic classification of smooth muscle tumors are based on the Stanford Criteria, and they are diagnosed by an assessment for abundant mitosis ( $\geq 10$ per $10 \mathrm{HPFs}$ ), cellular atypia and presence of areas of coagulative tumor cell necrosis. ${ }^{3,8}$ STUMP has a combination of the above features, without fulfilling the diagnosis of leiomyosarcoma. ${ }^{3}$ Given the heterogeneity of the histologic features of tumors classified in this group, it is conceivable that there is significant variation in the natural history and outcomes in this group. As such, multiple studies have further subclassified these tumors in an attempt to better predict prognosis. In this case, our patient had histologic features consistent with a diagnosis of STUMP as she had a low mitotic index and no coagulative necrosis, but diffuse significant cellular atypia. One such study further classified these tumors into "atypical leiomyoma with limited experience", "smooth muscle tumors of low malignant potential", "atypical leiomyoma - low risk of recurrence", and "mitotically active leiomyoma - limited experience". ${ }^{9}$ In this observational study, recurrence occurred in 2 of 16 cases, with both being in the "atypical leiomyoma with limited experience group" with histologic features of "multifocal moderate-tosevere atypia, no tumor cell necrosis and mitotic counts of four and five mitotic figures/10 HPFs, respectively", ${ }^{9}$ as is the case with our patient. It has been suggested that higher recurrence rates could be associated with diffuse immunoreactivity for p16 and P53. ${ }^{9,10}$ However, this should be used cautiously as the study numbers were small and need further evaluation before definitive outcomes can be drawn.

In general, these tumors are often slow growing and metastasize later when compared to leiomyosarcomas. ${ }^{3}$ Nevertheless, STUMP has been shown to recur, metastasize, and transform to leiomyosarcoma. ${ }^{11}$ Of note, it has been suggested that younger patients are more likely than their older counterparts to have a recurrence of disease. ${ }^{1}$ One study reported the average time for recurrence was 51 months, with recurrence recorded from 15 months to 9 years. ${ }^{12}$ The recurrence rate for STUMP is between $7.3 \%$ and $26 \%$ depending on the subtype, ${ }^{3,4,9,13}$ with an overall recurrence rate of $11 \% .^{3}$ However, true recurrence rates are difficult to determine, given the differing diagnostic criteria provided in studies, limited sample size and variable follow-up time periods provided in these studies. Of note, it has been suggested that tumors associated with recurrence often have a diffuse immunoreactivity of $\mathrm{p} 16$ and $\mathrm{p} 53 .^{3}$

Due to the paucity of literature available, a consensus regarding management has not been reached and clinicians are required to model further management and follow-up on limited, largely observational data. It has been suggested that the treatment of choice is a hysterectomy. The standard follow-up post-hysterectomy was six monthly for 5 years followed by yearly follow-up for a further 5 years with symptom review at each appointment and yearly imaging by MRI for evidence of recurrence or metastasis.

For patients who opt for fertility preservation, more frequent monitoring is required ${ }^{3}$ and needs to be weighed against the risk of recurrence. It is recommended that these patients should be evaluated for recurrence prior to conception. ${ }^{12}$ This is particularly important in this patient, given her histologic features, as she likely has an increased risk of recurrence of disease when compared to other forms of STUMP. While recurrence of disease often carries a good prognosis, death has been previously reported. ${ }^{12}$ Adjuvant therapy with progesterone, GnRH analog or chemotherapy has been suggested for patients who suffer from a recurrence of STUMP. ${ }^{1,3}$ However, little evidence is available regarding the use of these agents to prevent disease recurrence or in the management of primary disease. Our patient was initially treated with goserelin (GnRH agonist), which reduced the size of the STUMP tumor. Further research should be considered to investigate this agent's role in the management of this neoplasm.

Successful pregnancy has previously been documented after a preconception STUMP diagnosis. ${ }^{12}$ This occurred in a patient with p16- and 53-negative histopathology and spontaneous conception 6 months post-myomectomy. In this case, the patient underwent a hysterectomy at 1 year postpartum, and currently has no recurrence at 4 years postsurgery. This is of particular importance in this case as our patient is a 20 -year-old nulliparous woman and definitive management with hysterectomy could have significant psychological and psychosocial consequences with no clear improvement in prognosis. However, this needs to be balanced against her risk of recurrence and stringent follow-up is recommended with consideration of hysterectomy postnatally. Our patient has undergone extensive postoperative counseling and, with assistance from the Gynecology Oncology multidisciplinary team, has opted for fertility preservation and expectant management. 
All information pertaining to the aforementioned case has been collected through the PubMed search engine via keyword searches and use of Medical Subject Headings. Journal articles were excluded if the publication was not provided in English language text.

\section{Disclosure}

The authors report no conflicts of interest in this work.

\section{References}

1. Dall'Asta A, Gizzo S, Musaro A, et al. Uterine smooth muscle tumors of uncertain malignant potential (STUMP): pathology, follow-up and recurrence. Int J Clin Exp Pathol. 2014;7(11):8136-8142.

2. Picerno TM, Wasson MN, Gonzalez Rios AR, et al. Morcellation and the incidence of occult uterine malignancy: a dual-institution review. Int J Gynecol Cancer. 2016;26(1):149-155.

3. Ip PP, Tse KY, Tam KF. Uterine smooth muscle tumors other than the ordinary leiomyomas and leiomyosarcomas: a review of selected variants with emphasis on recent advances and unusual morphology that may cause concern for malignancy. Adv Anat Pathol. 2010;17(2): 91-112.

4. Guntupalli SR, Ramirez PT, Anderson ML, Milam MR, Bodurka DC, Malpica A. Uterine smooth muscle tumor of uncertain malignant potential: a retrospective analysis. Gynecol Oncol. 2009;113(3): 324-326.
5. Schwartz LB, Zawin M, Carcangiu ML, Lange R, McCarthy S. Does pelvic magnetic resonance imaging differentiate among the histologic subtypes of uterine leiomyomata? Fertil Steril. 1998;70(3):580-587.

6. Benson $\mathrm{C}$, Miah AB. Uterine sarcoma - current perspectives. Int J Womens Health. 2017;9:597-606.

7. Skorstad M, Kent A, Lieng M. Preoperative evaluation in women with uterine leiomyosarcoma. A nationwide cohort study. Acta Obstet Gynecol Scand. 2016;95(11):1228-1234.

8. Stewart EA, Quade BJ, Laughlin-Tommaso SK [homepage on the Internet]. Variants of uterine leimyomas (fibroids). 2017. Available from: http://www.uptodate.com. Accessed August 13, 2017.

9. Ip PP, Cheung AN, Clement PB. Uterine smooth muscle tumors of uncertain malignant potential (STUMP): a clinicopathologic analysis of 16 cases. Am J Surg Pathol. 2009;33(7):992-1005.

10. Atkins KA, Arronte N, Darus CJ, Rice LW. The use of p16 in enhancing the histologic classification of uterine smooth muscle tumors. Am J Surg Pathol. 2008;32(1):98-102.

11. Maccio A, Chiappe G, Kotsonis P, et al. Abdominal leiomyosarcomatosis after surgery with external morcellation for occult smooth muscle tumors of uncertain malignant potential: a case report. Int J Surg Case Rep. 2017;38:107-110.

12. Campbell JE, Knudtson JF, Valente PT, Robinson RD, Kost ER. Successful pregnancy following myomectomy for uterine smooth muscle tumor of uncertain malignant potential: a case report and review of the literature. Gynecol Oncol Rep. 2016;15:1-3.

13. Amant F, Moerman P, Vergote I. Report of an unusual problematic uterine smooth muscle neoplasm, emphasizing the prognostic importance of coagulative tumor cell necrosis. Int J Gynecol Cancer. 2005;15(6): 1210-1212.
International Journal of Women's Health

\section{Publish your work in this journal}

The International Journal of Women's Health is an international, peerreviewed open-access journal publishing original research, reports, editorials, reviews and commentaries on all aspects of women's healthcare including gynecology, obstetrics, and breast cancer. The manuscript management system is completely online and includes

\section{Dovepress}

a very quick and fair peer-review system, which is all easy to use. Visit http://www.dovepress.com/testimonials.php to read real quotes from published authors. 